\title{
Perceiving transformed movements when using tools
}

\author{
Christine Sutter $\cdot$ Sandra Sülzenbrück
}

Published online: 29 March 2012

(C) Springer-Verlag 2012

In the course of their evolution, humans have developed highly sophisticated skills in using tools to interact with their environment. When we use a tool (e.g., a hammer or a computer mouse), the segmental chain is extended to an object which is not a part of our body. As a consequence, the end effector is now the tip of the tool (the hammer head or the cursor on a monitor) and the movements of our body (mostly our hands) have to be adjusted so that we can execute precise movements with the tool. Therefore, the transformation between the required input (bodily movement) and the desired output (perceived movement of the effective part of the tool) has to be known and inverted to compute the appropriate bodily movement to perform a successful action with a tool (e.g., Greenwald 1970; Hommel et al. 2001; Wolpert and Flanagan 2010). Humans are remarkably adept at using tools with a broad range of complexity in the transformations required between movements of the body and movements of the tool (for an overview, see Sutter et al. in press). Whether we are using "classic" tools like a hammer or pliers or are mastering the complex transformations that are required, for example, for undertaking modern minimally invasive surgery-after more or less

This paper represents the editorial introduction to a Special Issue of the same name, guest-edited by the authors of this paper.

C. Sutter $(\bowtie)$

Department of Work and Cognitive Psychology,

RWTH Aachen University, Jägerstraße 17-19,

52056 Aachen, Germany

e-mail: Christine.Sutter@psych.rwth-aachen.de

S. Sülzenbrück

IfADo, Leibniz Research Centre for Working Environment and Human Factors, Ardeystraße 67, 44139 Dortmund, Germany

e-mail: suelzenbrueck@ifado.de practice, we (mostly) succeed. The aim of this Special Issue of Experimental Brain Research is to foster the understanding of processes involved in enabling human tool use and to provide an insight into basic mechanisms regarded as prerequisites for the skillful use of tools. Experts from different disciplines, for example, psychology, neuropsychology, medical sciences, applied medical engineering, and sport sciences address basic research questions and applied issues, and present current trends in research on human tool use.

The successful use of tools is often related to the acquisition of an internal representation of the encountered tool characteristics (e.g., sensorimotor transformation, familiarity and functionality of tool features). A number of studies in this Special Issue relate to the questions of how tools and their transformations are cognitively represented: For example, Macuga et al. (2012, this issue) investigate to what extent the dynamic features of a tool are incorporated into the internal representation of pointing movements. The authors assessed whether Fitts' law (1954) holds for motor imagery and the actual execution of pointing movements with three tools: a regular pen, a top-heavy pen and a bottom-heavy pen. Real and imagined movements with each tool followed the speed-accuracy relation described by Fitts' law, indicating that imagery and execution share internal representations. However, the knowledge about tool characteristics was more precisely represented in real than in imagined movements. It seems that differences in dynamic tool features are difficult to imagine and that the acquisition of specific tool knowledge is beyond motor imagery.

Knowledge about the object functionality and action semantics is another important prerequisite for the mastery of tools. In the study by Paulus et al. (2012, this issue), motor imagery as a training technique for the acquisition of novel functional object representations is investigated. The 
authors presented novel objects along with information about their functional use (required grip and goal location). In the training phase, participants were asked to imagine using the object in the designated way. The following retrieval phase showed that participants indeed acquired knowledge about the functional goal of the object, but not about the functional grip. In this respect, training by motor imagery resembles first-hand action training. Van Elk et al. (2012, this issue) present data from an fMRI study assessing the role of object familiarity for the planning of prospective actions with it. Participants imagined planning an action with either familiar (e.g., wine bottle) or unfamiliar objects (e.g., sports bottle). An action cue indicated the required action to be planned, that is, grasping the bottle to pour a drink or to stack it in a bucket/holder, or grasping the bottle at the upper or lower side. Action planning was faster for familiar than for unfamiliar objects. Neural activation increased in the anterior prefrontal brain areas for familiar objects, while it increased in parietal brain areas for unfamiliar objects. The authors conclude that action semantics of familiar objects are retrieved efficiently and effortlessly as compared to unfamiliar objects, especially when action planning is goal-directed. The study by Hermsdörfer et al. (2012, this issue) furthers the understanding of representation and retrieval of action semantics. They report data from healthy participants as well as patients with left or right brain damage following stroke, comparing the characteristics of different modes of action execution associated with tool use. Left brain damage is particularly associated with apraxia, which in turn impairs the patient's ability to pantomime tool-use actions. The authors studied the kinematic characteristics of pantomiming, demonstrating and the actual usage of a ladle in repetitive scooping movements. Although in healthy subjects, both pantomime and demonstration differed from the actual use, kinematics of hand movements after left brain damage revealed similar patterns in all three modes of action execution, with the most severe impairment found in the scooping component of the task. From these findings, the authors conclude that common motor schemas are activated for a specific tool-use action. These schemas can be adjusted to the requirements of the actual task in healthy subjects. However, left brain lesions are associated with a generalized deficit across different modes of action execution.

The contribution by Masaki et al. (2012, this issue) investigates timing control that becomes important when using tools in order to control moving objects. For instance, hitting a baseball with a bat requires a precise temporal anticipation of both sensory input and motor output as well as close performance monitoring. The EEG study examined the neural mechanisms associated with timing control in a coincident timing task. The task was to press a force-sensitive key such that a specified peak force was reached when the (slow or fast) moving stimulus crossed a defined position. For more ballistic movements (having a short time-topeak force interval) and rapidly moving stimuli, absolute timing errors were smaller. These results indicate that for a precise timing control, more ballistic movements are advantageous even for rapidly moving stimuli. The analysis of the EEG data showed that the amplitudes of the contingent negative variation decreased for slow moving stimuli and for more ballistic movements. This possibly reflects less effort in preprogramming responses. The motor-elicited negativity was largest for more ballistic movements with slow stimuli, probably representing processes related to performance monitoring and motor output.

The impact of knowledge about the action's goal or the tool and its transformation was studied by Massen and Sattler (2012, this issue). Presenting advance information concerning the spatial goal of the action and the tool transformation affected efficiency of programming a tool-use action. If tool-use actions are represented by their goals, then advance information about the goal should facilitate performance, and vice versa, if they are represented in terms of the tool and its transformation. In response to a spatial or symbolic cue, targets had to be touched with two levers simultaneously operated by the left and right hands. Bimanual tool actions varied with respect to congruency of tool transformation and congruency of target direction. Results showed that having advance information about the tool transformation resulted in more efficient action programming than having advance information about the spatial target. The authors conclude that bimanual tool-use actions are directed by the representations of the transformation of the tool rather than being goal-directed. Dietrich et al. (2012, this issue) investigate how tool transformations influence the unimanual coordination of movements with external events. In their study they investigated whether this coordination with an external event is related to the hand movement (proprioceptive/tactile feedback) or to the visual feedback of the movement displayed on a monitor. The pattern of performance differences in a circle drawing task with different display configurations and different types of visual feedback indicates that the distal effects dominate unimanual action coordination with external events. The understanding of how the human information processing system deals with diverse proprioceptive/tactile and visual feedback is furthered by Sutter and Ladwig (2012, this issue). They did not use a tool, but visual feedback of stimuli and hand movements was spatially inverted (left-right transformation) in one condition as if a tool transformation similar to a two-sided lever (cf. Sülzenbrück and Heuer 2012, as well as Massen and Sattler 2012, in this Special Issue) had been in effect. Performing aiming movements in response to colored stimuli while the spatial location of the stimulus had to be ignored resulted in a Simon effect when 
visual and proprioceptive/tactile feedback corresponded (no transformation), and eliminated or even inverted the Simon effect with opposing visual and proprioceptive/tactile feedback (spatial transformation). Impairing afferent information so that proprioceptive/tactile feedback from the moving hand was no longer a reliable source for planning and controlling actions attenuated compatibility effects, and preserved the Simon effect across all feedback conditions. Multisensory integration followed the "ideal observer" model (e.g., Ernst and Banks 2002): The most reliable information from a sensory system is prioritized and therefore gains dominance in planning and controlling actions.

The contributions by Cardinali et al. (2012, this issue) and Holmes (2012, this issue) provide an overview about the plasticity of the human brain, and the conditions and constraints of its capability of incorporating tools into body representations for action. On the one hand, Cardinali et al. (2012, this issue) present findings from perceptual and motor consequences of tool use-such as changes in space representation, in the localization of somatosensory stimuli on the body surface and in the kinematics of hand movements during and after tool use-which support the notion that tools seem to be somehow and at least temporarily "incorporated" into body representations (e.g., Iriki et al. 1996; Ladwig et al. 2012). Holmes, on the other hand, puts this ongoing discussion on tools becoming extensions of the human body into question by providing evidence for and against this hypothesis from neurophysiological, neuropsychological and behavioral fields. He elaborates the latter aspect in a meta-analysis of 7 single studies (total $N=180$ ) from his laboratory using a crossmodal congruency task to investigate how tool use modulates body representations. The results indicate that - at least in a crossmodal congruency task-tool use does not literally extend peripersonal space.

A further series of studies relate to the question of how humans acquire cognitive representations of tools and which neural structures contribute to this process. Sülzenbrück and Heuer (2012, this issue) investigate the effect of enhanced mechanical transparency on the acquisition of an internal representation of a tool transformation. In their study, participants practiced moving the tip of the load arm of a lever to different target positions by moving the tip of the effort arm with their hand. While two experimental groups only saw a cursor reflecting the tip of the load arm on the monitor, a third group saw representations of the tip as well as the load arm on the monitor throughout each movement. While during practice the visible lever arm enhanced performance in terms of straighter and faster movement, participants who had practiced with the visible lever arm showed less accurate internal representations of the visuomotor transformation in subsequent test trials without visual feedback. These findings support the notion that the acquisition of an accurate internal representation of a tool transformation becomes less likely that the more feedback control is possible during practice (for a review, see Sülzenbrück 2012). The study conducted by Leow et al. (2012, this issue) also addresses the motor learning processes enabling skilled tool use. Although the adaptation to novel sensorimotor transformations encountered during tool use is often associated with the acquisition of an internal model of the tool transformation (Wolpert and Kawato 1998), recent findings indicate that also model-free approaches like operant reinforcement learning are involved in sensorimotor adaptation (Huang et al. 2011). Reinforcement learning is associated with activity in the basal ganglia and should therefore be impaired in patients with Parkinson's disease (PD). In their study, Leow et al. compared savings, which is a faster relearning of previously learned motor adaptations, between PD patients and healthy elderly participants. The finding of impaired savings in PD patients after learning a visuomotor rotation indicates that basal ganglia are involved in motor adaptation. The authors argue that the malfunctioning striatal dopaminergic signaling associated with PD impairs the modulation of dopaminergic signals to the motor cortex in response to rewarding motor outcomes. This in turn inhibits the association between the motor command and the reward of their outcome.

For successful tool use, a variety of competencies have to be acquired during human development, with their fundamentals being shaped during early childhood. Disselhorst-Klug et al. (2012, this issue) introduce an objective method to evaluate the spontaneous motor activity development with increasing age in infants. Within the first 6 months of a newborn's life, spontaneous motor activity changes from writhing movements, to fidgety movements and finally to voluntary movements (linguistic approach by Prechtl 2001). Disselhorst-Klug et al. analyze 3D recorded movement trajectories of healthy infants and infants with infantile cerebral palsy. They define specific movement parameters in accordance with Prechtl's categories. This quantitative approach provides an objective description of age-appropriate developmental changes in motor activity. Since this quantitative analysis is sensitive toward pathologies in motor development, it shows how infants learn to interact with their environment from the very beginning of their life. Kahrs et al. (2012, this issue) further the understanding of how early progresses in motor activity transition to an instrumental and more controlled motor action. Therefore, they analyzed the development of infant's banging movements as a type of repetitive action preparing for tool use. For 7- to 14-month-old infants hand trajectories become more efficient and goal-directed with age. It can be concluded that developmental changes in spontaneous (banging) movements contribute to the coordination of 
perception and action - a crucial precondition for the use of tools.

The research presented by Caçola and Gabbard (2012, this issue) investigates developmental aspects of space representations. Even young infants have spatial representations of peripersonal space (within grasp area) and perceive that near space is extendable to accommodate reachability: For instance, by 8 months of age, infants lean forward to extend the range of contact, by 12 months they use a tool (e.g., a rod) to reach something that is initially out of reach (McKenzie et al. 1993). The study by Caçola and Gabbard is based on the assumption that peripersonal and extrapersonal (beyond reach) space needs to be constantly recalibrated during childhood due to changes in body size and relative relations of limbs. Furthermore, space representations need to be at least temporally modified when using tools (for an overview on body schema and space representation, see Cardinali et al. as well as Holmes in this Special Issue). As a consequence, children, but not adults, should have difficulties mapping extrapersonal space, especially when using a tool. In their study, children between 7 and 11 years of age and young adults estimated the reachability of targets within and without grasp range while holding or not holding a stick. Performance between younger and older children did not differ, but estimates were less accurate as compared with those of adults. Additionally, tool length influenced estimations of reach. Osiurak et al. (2012, this issue) follow this line of research, but contrary to Caçola and Gabbard, they did not explicitly instruct participants (to imagine) using the tool when estimating the reachability of targets appearing in extrapersonal space. Nevertheless, participants underestimated the distances only when holding a baton with which they could have reached the targets as compared to when not holding it. These findings demonstrate a spontaneous and implicit intention for humans to use tools if these substantially extend their action capabilities.

To sum up, when using tools a major challenge for the human information processing system is providing a high degree of flexibility to compensate for and adapt to changes in the environment. The studies included in this Special Issue reflect the diverse approaches of the latest developments in research on human tool use. These contributions seek to continue the ongoing trend to advance the understanding of tool-use behavior as well as its basic motor and cognitive mechanisms (for a recent special issue of the Journal of Psychology, see Massen and Rieger 2012).

Acknowledgments We are very grateful for the support of Margrit Derrick, Mel Goodale and Neil Solomon, and the reviewers in composing this Special Issue.

\section{References}

Caçola P, Gabbard C (2012) Modulating peripersonal and extrapersonal reach space via tool use: a comparison between 6- to 12-yearolds and young adults. Exp Brain Res (this issue). doi:10.1007/ s00221-012-3017-8

Cardinali L, Jacobs S, Brozzoli C, Frassinetti F, Roy AC, Farnè A (2012) Grab an object with a tool and change your body: tool-usedependent changes of body representation for action. Exp Brain Res (this issue). doi:10.1007/s00221-012-3028-5

Dietrich S, Prinz W, Rieger M (2012) Vision-to-event and movementto-event coordination in an unimanual circling task. Exp Brain Res (this issue). doi:10.1007/s00221-012-3005-Z

Disselhorst-Klug C, Heinze F, Breitbach-Faller N, Schmitz-Rode T, Rau G (2012) Introduction of a method for quantitative evaluation of spontaneous motor activity development with age in infants. Exp Brain Res (this issue). doi:10.1007/s00221-012-3015-x

Ernst MO, Banks MS (2002) Humans integrate visual and haptic information in a statistically optimal fashion. Nature 415:429-433. doi: $10.1038 / 415429$ a

Fitts PM (1954) The information capacity of the human motor system in controlling the amplitude of movement. J Exp Psychol 47:381391

Greenwald AG (1970) Sensory feedback mechanisms in performance control: with special reference to the ideo-motor mechanism. Psychol Rev 77:73-99. doi:10.1037/h0028689

Hermsdörfer J, Li Y, Randerath J, Goldenberg G, Johannsen L (2012) Tool use without a tool: kinematic characteristics of pantomiming as compared to actual use and the effect of brain damage. Exp Brain Res (this issue). doi:10.1007/s00221-012-3021-z

Holmes NP (2012) Does tool use extend peripersonal space? A review and re-analysis. Exp Brain Res (this issue). doi: 10.1007/s00221012-3042-7

Hommel B, Müsseler J, Aschersleben G, Prinz W (2001) The Theory of Event Coding (TEC): a framework for perception and action planning. Behav Brain Sci 24:849-878. doi:10.1017/S0140525X 01000103

Huang V, Haith A, Mazzoni P, Krakauer J (2011) Rethinking motor learning and savings in adaptation paradigms: model-free memory for successful actions combines with internal models. Neuron 70:787-801. doi:10.1016/j.neuron.2011.04.012

Iriki A, Tanaka M, Iwamura Y (1996) Coding of modified body schema during tool use by macaque postcentral neurones. NeuroReport 7:2325-2330

Kahrs B, Jung W, Lockman J (2012) What is the role of infants' early banging in the development of tool use? Exp Brain Res (this issue). doi:10.1007/s00221-012-3062-3

Ladwig S, Sutter C, Müsseler J (2012) Crosstalk between proximal and distal action effects when using a tool. J Psychol 220:10-15. doi: 10.1027/2151-2604/a000085

Leow LA, Loftus AM, Hammond G (2012) Impaired savings despite intact initial learning of motor adaptation in Parkinson's disease. Exp Brain Res (this issue)

Macuga KL, Papailiou AP, Frey SH (2012) Motor imagery of tool use: relationship to actual use and adherence to Fitts' law across tasks. Exp Brain Res (this issue). doi: 10.1007/s00221-012-3004-0

Masaki H, Sommer W, Takasawa N, Yamazaki K (2012) Neural mechanisms of timing control in a coincident timing task. Exp Brain Res (this issue). doi:10.1007/s00221-012-3052-5

Massen C, Rieger M (2012) Editorial of the special issue: Cognitive and motor aspects of human tool use. J Psychol 220:1-2. doi:10.1027/2151-2604/a000083

Massen C, Sattler C (2012) What to do and how to do it: action representations in tool use. Exp Brain Res (this issue). doi:10.1007/ s00221-012-3003-1 
McKenzie BE, Skouteris H, Day RH, Hartman B, Yonas A (1993) Effective action by infants to contact objects by reaching and leaning. Child Dev 64:415-429. doi:10.1111/j.1467-8624.1993. tb02918.x

Osiurak F, Morgado N, Palluel-Germain R (2012) Tool use and perceived distance: when unreachable becomes spontaneously reachable. Exp Brain Res (this issue). doi:10.1007/s00221-012-3036-5

Paulus M, van Elk M, Bekkering H (2012) Acquiring functional object knowledge through motor imagery? Exp Brain Res (this issue)

Prechtl HFR (2001) General movement assessment as a method of developmental neurology: new paradigms and their consequences. Dev Med Child Neurol 43:836-842. doi:10.1111/ j.1469-8749.2001.tb00173.x

Sülzenbrück S (2012) The impact of continuous and terminal visual feedback on the mastery of visuo-motor transformations. J Psychol 220:3-9. doi:10.1027/2151-2604/a000084

Sülzenbrück S, Heuer H (2012) Enhanced mechanical transparency during practice impedes open-loop control of a complex tool. Exp Brain Res (this issue). doi:10.1007/s00221-012-3011-1
Sutter C, Ladwig S (2012) Mirrored visual feedback limits distal effect anticipation. Exp Brain Res (this issue). doi:10.1007/s00221-0123018-7

Sutter C, Sülzenbrück S, Rieger M, Müsseler J (in press) Limitations of distal effect anticipation when using tools. New Ideas Psychol van Elk M, Viswanathan S, van Schie HT, Bekkering H, Grafton ST (2012) Pouring or chilling a bottle of wine: an fMRI study on the prospective planning of object-directed actions. Exp Brain Res (this issue). doi:10.1007/s00221-012-3016-9

Wolpert DM, Flanagan JR (2010) Motor learning. Curr Biol 20:R467R472. doi:10.1016/j.cub.2010.04.035

Wolpert DM, Kawato M (1998) Multiple paired forward and inverse models for motor control. Neural Netw 11:1317-1329. doi:10.1016/S0893-6080(98)00066-5 